\title{
Perché la dialisi peritoneale in Italia ha una bassa penetrazione
}

\author{
Giuseppe La Greca
}

\author{
Divisione di Nefrologia, Dialisi e Trapianto \\ Ospedale "San Bortolo", Vicenza
}

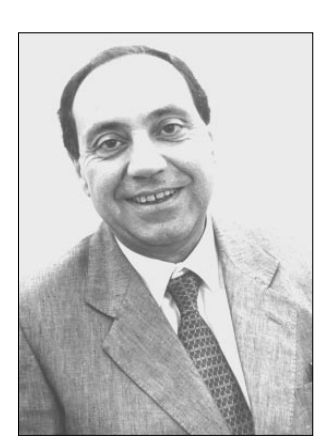

$\grave{\mathbf{E}}$ ormai acquisito, nella comunità nefrologica internazionale, che entro i primi cinque anni dall'inizio del trattamento sostitutivo, il tasso di mortalità dei pazienti trattati con dialisi peritoneale è inferiore o uguale - quando corretto per età, comorbidità e eziologia - a quello dei pazienti trattati con emodialisi (Schaubel et al, PDI, 1998).

A ciò si deve aggiungere una migliore preservazione della diuresi residua, un miglior controllo dell'ipertensione, della crasi ematica e la relativamente rara comparsa di insufficienza renale acuta post-trapianto in caso di trapianto renale.

Oltre a ciò il paziente in dialisi peritoneale esegue il suo trattamento al proprio domicilio e quindi non contribuisce ad affollare i centri di emodialisi extracorporea; la popolazione in dialisi peritoneale costituisce, purché la struttura di riferimento sia ben organizzata, un polmone facilmente espansibile e in grado di provvedere - senza incrementare le strutture fisse - al continuo e progressivo incremento dei pazienti che necessitano della terapia dialitica.

Tuttavia, nonostante questi dati siano largamente conosciuti nella comunità nefrologica, da anni assistiamo in Italia a una stagnazione (intorno ai valori del $10 \%$ della popolazione dialitica) dei pazienti trattati con dialisi peritoneale.

Questo fenomeno, come si evince dalla Tabella I non è tipicamente italiano; infatti in Europa il valore medio della popolazione prevalente in
D.P è del 12.5\% (range UK 37.4 Portogallo 5.2).

Se consideriamo l'utilizzo della dialisi peritoneale in Italia dove a fronte di un lento e progressivo incremento della popolazione in trattamento dialitico, la proporzione dei pazienti trattati con dialisi peritoneale si mantiene stabilmente intorno al $10 \%$ (Tab. II).

L'Italia è un Paese in cui il fabbisogno dialitico può considerarsi piena-

TABELLA I - PAZIENTI TOTALI E \% IN PD IN EUROPA

\begin{tabular}{lrrr}
\hline Stato & Paz. Tot. & Paz. in PD & \% Paz.in PD \\
\hline Francia & 26271 & 2870 & 10.7 \\
\hline Belgio & 4316 & 393 & 9.1 \\
\hline Olanda & 4771 & 1390 & 31.1 \\
\hline U.K. & 16916 & 6326 & 37.4 \\
\hline Danimarca & 1964 & 546 & 27.8 \\
\hline Finlandia & 1086 & 297 & 27.4 \\
\hline Norvegia & 618 & 135 & 21.8 \\
\hline Svezia & 2836 & 729 & 25.7 \\
\hline Germania & 46699 & 2895 & 6.2 \\
\hline Italia & 41402 & 4140 & 10.0 \\
\hline Spagna & 18197 & 1447 & 9.6 \\
\hline Austria & 2845 & 245 & 12.0 \\
\hline Svizzera & 2336 & 280 & 20.8 \\
\hline Irlanda & 1315 & 273 & 5.2 \\
\hline Portogallo & 6812 & 354 & 11.5 \\
\hline Grecia & 6853 & 788 & 12.5 \\
\hline TotALE & 185237 & 23108 &
\end{tabular}


mente soddisfatto: popolazione incidente > 130 pazienti/milione/anno; popolazione prevalente $>770$ pazienti/milione/ cui vanno aggiunti 160 pazienti/milione con trapianto di rene funzionante.

La rete italiana dei servizi di dialisi comprende 875 Centri (15.2/milione abit.) di cui 609 pubblici e 266 privati. I Centri pubblici si suddividono in Centri di emodialisi ad assistenza limitata (CAL) e servizi di nefrologia in gran parte dotati di posti letto per le degenze nefrologiche e associati al Centro di emodialisi/ dialisi peritoneale; questi Centri sono stati da noi definiti come Centri madre (CM).

Disaggregando i dati e suddividendo l'Italia in tre macroregioni (Tab. III) si possono rilevare dati di grande interesse. Passando da Nord a Sud si osserva una riduzione del numero dei CAL e un parallelo incremento dei Centri privati con un rapporto tra Centri pubblici e Centri privati che passa dal $96.2 \%$ del Nord, all' $81.9 \%$ del Centro e arriva al

TABELLA II - LA DIALISI IN ITALIA

POPOLAZIONE
CENTRI DIALISI
CENTRI DIALISI PUBBLICI
CENTRI DIALISI PRIVATI
RAPPORTO PUBBLICO / PRIVATO
TOTALE PAZIENTI
TOTALE PAZIENTI PD - 4.140
PAZIENTI PD / PAZIENTI TOT.
entro madre; servizio autonomo di Nefrologia e/o Dialisi

$875=15.2 / 10^{6}$ abitanti 609

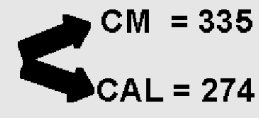

266

$69.60 \%$

CAPD $70 \%$ 41.402

APD $30 \%$

$\mathrm{CM}$ = Centro madre; servizio autonomo di Nefrologia e/o Dialisi

$\mathrm{CAL}=$ Centro ad assistenza limitata

$\mathrm{CAPD}=$ Continuous Ambulatory Peritoneal Dialysis

$\mathrm{APD}=$ Automated Peritoneal Dialysis

( Aggiornamento al 30.06.2000)

TABELLA III - LA DIALISI IN ITALIA

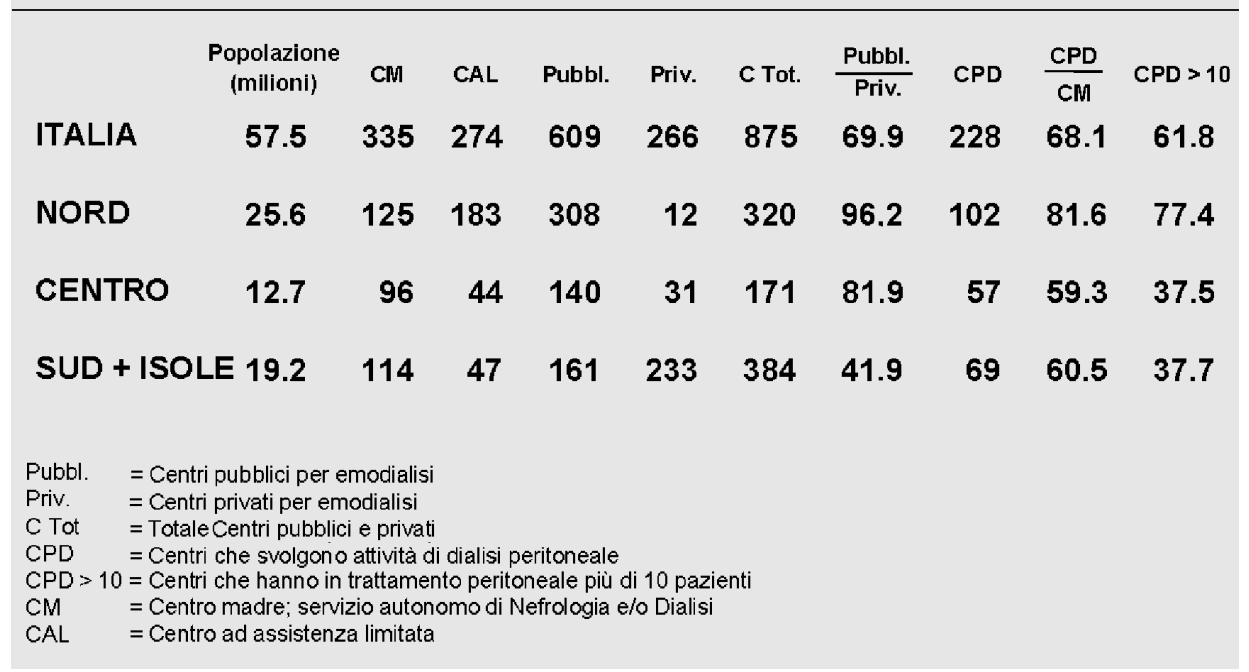

$41.9 \%$ nell'Italia meridionale e insulare.

Quanto alla distribuzione dei Centri sul territorio nazionale in rapporto all'entità della popolazione, si può rilevare che al Nord vi sono 12.5 Centri per milione di abitanti, nell' Italia centrale tale numero sale a 13.4 /milione e arriva a $20 /$ milione nel Sud e nelle isole maggiori. Ciò è dovuto, come si può evincere dalla Tabella III, all'incremento, da Nord a Sud dei Centri dialisi privati ( 223 nel Sud) e comporta una suddivisione della rete dialitica in numerosi piccoli Centri spesso scollegati da Centri di riferimento nefrologici dove appoggiare i pazienti in caso di necessità.

Per quanto attiene la dialisi peritoneale va rilevato che questa non viene praticata nei CAL perché, laddove esista al Centro di riferimento (CM) un programma di trattamento con peritoneale i pazienti vengono gestiti centralmente, e tantomeno nei Centri dialisi privati sia perché il rimborso relativo offerto dal SSN non è remunerativo in confronto all'emodialisi, sia perché un programma di dialisi peritoneale deve poter contare su un Centro di nefrologia attrezzato e operativo $24 \mathrm{~h} /$ die dove appoggiare i pazienti che presentano problemi clinici.

Pertanto, alla luce di queste considerazioni, solo i Centri pubblici/autonomi di nefrologia e dialisi - in Italia questi assommano a 335 - possono sviluppare un programma di dialisi peritoneale (Tab. IV). Di questi, 228 pari al $68 \%$ del totale, offrono ai loro pazienti l'opportunità di un trattamento con dialisi peritoneale ma, molti Centri hanno in trattamento pochi pazienti, verosimilmente per la scarsa convinzione dei loro nefrologi della validità del trattamento e dell'opportunità di praticarlo.

Pertanto abbiamo arbitrariamente considerato operativi quei Centri che hanno in trattamento più di 10 pazienti e abbiamo constatato che solo $141(61.8 \%)$ dei Centri che fanno dialisi peritoneale superano il limite di 10 pazienti. Se poi si considera la distribuzione in Italia di questi Centri si può osservare che la 
diffusione della dialisi peritoneale (CPD/CM) diminuisce dal Nord al Sud con proporzionalità inversa rispetto al numero dei Centri privati e che, sia nel Centro che nel Sud Italia, la percentuale dei Centri che fanno dialisi peritoneale e di quelli che hanno in trattamento più di 10 pazienti è nettamente inferiore rispetto al Nord.

Per rendere ancora più evidenti queste differenze abbiamo, arbitrariamente, preso in considerazione (Tab. V) 3 Regioni del Nord e 3 Regioni del Centro Sud comparabili per popolazione residente. Si può rilevare che mentre al Nord vi è una notevole diffusione di CAL e un rapporto Centri pubblici/Centri privati di circa 96\%, nelle 3 Regioni del Centro Sud solo il $26.5 \%$ dei Centri sono pubblici e i Centri privati assommano a 241. Parallelamente, per quanto attiene la dialisi peritoneale, vi sono al Sud meno Centri che la offrono ai loro pazienti (circa la metà dei CM) e i Centri operativi con più di 10 pazienti in trattamento, valutati in termini percentuali, sono significativamente meno al Sud $(77.6 \%$ vs $29.7 \%$ ).

Tutto ciò conferma l'impressione che laddove vi è un massiccio sviluppo dei Centri privati, e conseguentemente dell'emodialisi "profit", vi sono scarse possibilità di fare crescere la dialisi peritoneale.

\section{Conclusioni}

Pur in assenza di studi prospettici, controllati e randomizzati si deve oggi ritenere, sulla base di studi osservazionali, sia prospettici che retrospettivi, che non vi sono, entro i primi cinque anni di terapia sostitutiva, significative differenze, in termini di sopravvivenza dei pazienti tra emodialisi e dialisi peritoneale. Ciò è ulteriormente confermato anche da un recentissimo studio multicentrico, prospettico, non randomizzato, condotto in Canada su 822 pazienti consecutivi trattati in 11 Centri canadesi (Murphy et al, Kid Int 2000; 57: 1720).

Tuttavia, nonostante le evidenze scientifiche riportate suggeriscano un più largo impiego della dialisi peritonale, lo sviluppo di questa tecnica che da alcuni anni ci si sarebbe aspettato non si è ancora verificato e gli indici di prevalenza, in Europa e in Italia, sono pressoché stazionari.

Il problema può essere esaminato con approcci diversi: quello eticoculturale, economico-organizzativo e normativo.

Ci sembra eticamente scorretto non proporre ai pazienti, come prima scelta, un trattamento che, almeno per i primi cinque anni, offre le stesse probabilità di sopravvivenza del- l'emodialisi e che nella maggior parte dei casi permette una riabilitazione e una qualità di vita superiori. Oltre alla CAPD, l'utilizzo delle diverse e più recenti tecniche di APD consente una flessibilità del trattamento che può essere modulata in rapporto alle esigenze lavorative $\mathrm{e}$ relazionali di ciascun paziente. Pertanto, si dovrebbe fare in modo che il paziente, edotto correttamente dei vantaggi e dei limiti di ciascuna tecnica, possa esercitare una libera opzione tra il trattamento intra- ed extracorporeo. Fondamentale comunque è il fatto che i componenti del-

TABELLA IV - DIALISI PERITONEALE IN ITALIA

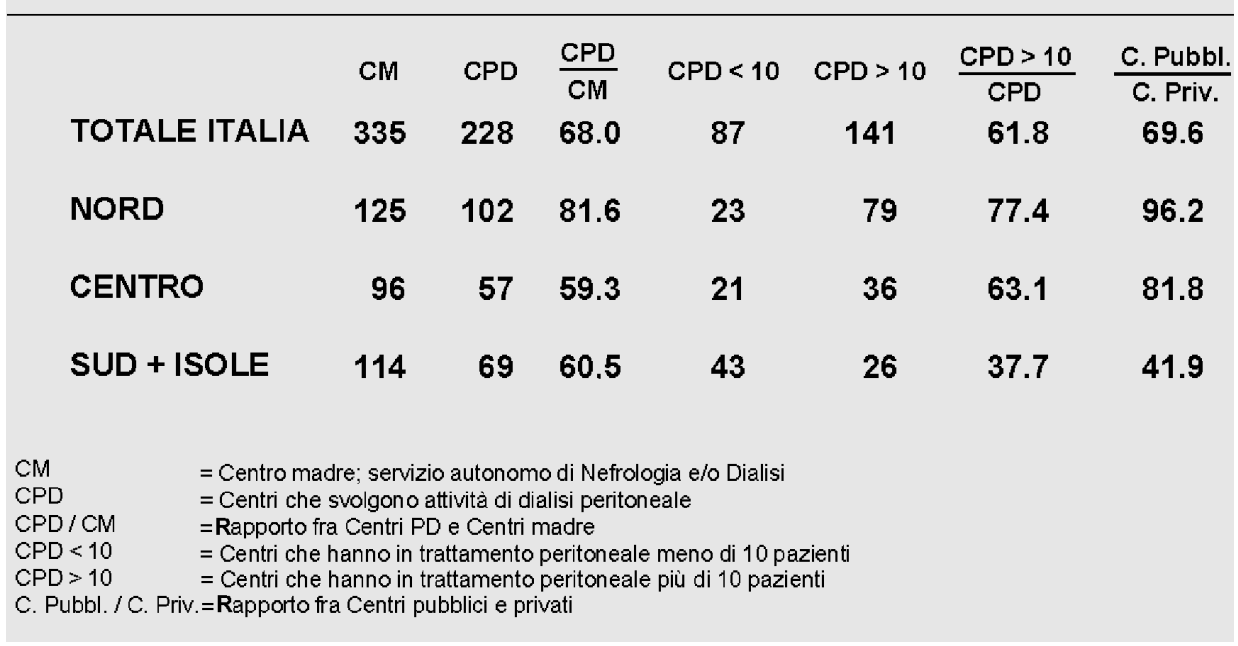

TABELLA V - LA DIALISI IN ITALIA - REGIONI A CONFRONTO

\begin{tabular}{lrrrrrrrrrr} 
& $\begin{array}{c}\text { Popolazione } \\
\text { (milioni) }\end{array}$ & CM & CAL & Pubbl. & Priv. & C Tot. & $\frac{\text { Pubbl. }}{\text { Priv. }}$ & CPD & $\frac{1}{C}$ CPD & CPD > 10 \\
Piemonte-V.A. & 4.41 & 23 & 29 & 52 & 0 & 52 & 100.0 & 22 & 95.6 & 72.7 \\
Lombardia & 9.02 & 40 & 63 & 103 & 8 & 111 & 92.7 & 38 & 95.0 & 89.5 \\
Veneto & 4.49 & 22 & 22 & 44 & 0 & 44 & 100.0 & 16 & 72.7 & 56.2 \\
\hline Totale & 17.92 & 85 & 114 & 199 & 8 & 207 & 96.1 & 76 & 89.4 & 77.6 \\
\hline Lazio & 5.25 & 25 & 8 & 33 & 28 & 61 & 54.1 & 12 & 48.0 & 50.0 \\
Campania & 5.80 & 23 & 3 & 26 & 133 & 159 & 16.3 & 11 & 47.8 & 9.1 \\
Sicilia & 5.10 & 25 & 3 & 28 & 80 & 108 & 25.9 & 14 & 56.0 & 28.5 \\
\hline Totale & 16.15 & 73 & 14 & 87 & 241 & 328 & 26.5 & 37 & 50.7 & 29.7 \\
\hline
\end{tabular}


l'équipe proponente l'opzione e i medici in particolare, siano intimamente convinti di offrire la scelta tra due metodiche equivalenti e di pari dignità.

Per quanto attiene gli aspetti economici e organizzativi, senza entrare nel merito dei costi dei materiali dialitici (soluzioni, filtri, aghi fistola ecc.), si deve tener conto del fatto che disponendo di un programma di dialisi peritoneale efficiente si risparmia l'aumento a dismisura delle strutture fisse per emodialisi o la necessità di ricorrere a tripli e talora quadrupli turni giornalieri su ogni posto dialisi come avviene in taluni Centri dialisi per far fronte all'immissione di nuovi pazienti. Non secondario appare il risparmio del personale infermieristico che, oltre a costare più del $50 \%$ della spesa totale dell'emodialisi, comincia a scarseggiare in maniera significativa. A chiarimento di ciò valga l'esempio del nostro Centro dove due medici e quattro infermieri sono sufficienti a gestire un programma di dialisi peritoneale con ottanta pazienti mentre per un pari numero di pazienti in emodialisi sono necessari tre medici e venti infermieri.

Da quanto detto appare chiaro che un programma integrato di dialisi peritoneale ed extracorporea rende il Centro che lo attua flessibile e in grado di accogliere e trattare adeguatamente tutti i pazienti che necessitano di terapia sostitutiva renale, sia in vista di un eventuale trapianto renale che per un trattamento dialitico cronico.

Di non minore importanza appaiono gli aspetti normativi che attualmente, in Italia, non favoriscono l'utilizzo della metodica. Infatti il rimborso che il SSN paga all'azienda ospedaliera per la dialisi peritoneale è mediamente inferiore a quello dell'emodialisi, senza tener conto che l'impegno dei medici per seguire un gruppo di pazienti che si dializzano al proprio domicilio è mediamente superiore a quello dedicato a un pari gruppo di pazienti in emodialisi.

Se è pur vero che il tasso di ospedalizzazione e quindi di costi indotti dei pazienti in peritoneale è superiore a quello dell'emodialisi, non riteniamo che i criteri di scelta debbano dipendere da una mera valutazione economica a breve termine ma che si debbano valutare il vantaggio clinico del paziente e il minor impatto logistico-organizzativo legati all'uso della peritoneale come prima scelta. È necessario pertanto che la Società Italiana di Nefrologia affronti il problema sia promuovendo tra i nefrologi italiani la cultura della dialisi peritoneale sia agendo con adeguate e corrette informazioni sulle autorità politico-istituzionali del Ministero della Sanità e degli Assessorati Regionali alla Sanità in modo da rendere maggiormente diffuso e operante il programma integrato dialisi peritoneale/emodialisi.

nefrologia@goldnet.it 